\title{
FATIGUE OF JACKET PLATFORMS: EFFECT OF MARINE GROWTH MODELLING
}

\author{
SCHOEFS Franck \\ GeM, Institut de Recherche en Génie Civil et \\ Mécanique, Université de Nantes-École Centrale de \\ Nantes-CNRS, Nantes, France
}

\author{
GUILLO Christophe \\ GeM, Institut de Recherche en Génie Civil et \\ Mécanique, Université de Nantes-École Centrale de \\ Nantes-CNRS, Nantes, France
}

\author{
BOUKINDA Morgan \\ GeM, Institut de Recherche en Génie Civil et Mécanique, \\ Université de Nantes-École Centrale de Nantes-CNRS, \\ Nantes, France
}

\author{
ROUHAN Antoine \\ Bureau Veritas \\ Courbevoie, France
}

\begin{abstract}
After decades of developments of methods and software, reassessment of jacket structures is still a challenge. This paper focus on marine growth effects in case of fatigue behavior for Jacket offshore structures. Two main items are pointed out: selection of marine growth profile and modeling of hydrodynamic coefficients in presence of marine growth. A complete approach is provided and completed with bot sensitivity and uncertainty studies.
\end{abstract}

\section{INTRODUCTION.}

Jacket structures are submitted to waves loading which directly depends on the marine growth profile. As it thickness, type, percentage cover etc. changes the forces on members, it also affect fatigue of tubular joints. Operators nowadays want to be able to demonstrate that actual marine growth profile of existing platforms do not affect significantly fatigue of tubular joints, reducing costly marine fouling cleaning campaigns. Also, frequency of inspection campaigns based on engineering computations may be affected.

In usual fatigue and push-over engineering computations, forces on members are computed by means of Morison's Formula. Marine growth is accounted for by means of hydrodynamic coefficient.

In design phase, such coefficients can be found in API-RP2A(WSD) recommendation [1]. They are based on the assumption that the Reynolds number is in the post-critical area.

However, for real structure with a different profile of marine growth than the one proposed in the API-RP-2A(WSD), hydrodynamic conditions show that Reynolds number may be in the critical or sub-critical regions. Thus, hydrodynamics coefficient differs.
In this paper, it is shown how these coefficients are computed. A semi-probabilistic approach, based on trajectories analysis of stochastic process for water particle velocity, is suggested. The impact on forces and fatigue computations are shown. Finally, as marine growth profiles are subject to changes within time, a sensitivity study on profiles is performed to show how the fatigue Miner damage of most critical tubular joints are affected. A fatigue sensitivity ratio to marine growth $(F R M)$ is defined and proposed as rational aidtool for inspection/cleaning intentions.

\section{METHODS OF REASSESSMENT OF STRUCTURES SUBMITTED TO MARINE GROWTH}

\section{Dynamic behavior}

Even if most of jacket platforms are non resonant structures, marine growth leads to an over-weight mainly placed on the top of the structures, where light and food are plentiful. Despite this fact, this problem has not be identified as dominant. For some components as risers, this question must be looked at.

\section{Quasi-static behavior}

Marine growth is the source of component diameter increasing and modify the hydrodynamics around component beams. Two approaches can be suggested:

- one concerns mainly sites where seasonal effects on marine growth colonization process are not of first importance. It is the case in areas located in Gulf of Guinea. Then a reasoning based on a reference period is suitable [2].

- the other one is well adapted when winter storms and other seasonal effects are significant. That is the 
case for sites in North Sea. Then an approach based on correlation between seasonal effects and storms occurrence must be introduced [3].

\section{Fatigue behavior}

Fatigue analyses are performed for various purposes, ringing from design checks to inspection planning. In the following, the fatigue design factor is used. It is defined as the ratio of fatigue life of the welded joint to the intended service life of the platform.

For design, the requirement from codes is typically that the fatigue design factor (FDF) do not exceed a given value for tubular joints [1][4]. The value of the FDF is important but not critical. To the contrary, inspection planning, using Risk Based Inspection for example [5][6][7], needs accurate FDF values. The inspection times are defined according to the FDF value. It is a key parameter in defining inspection frequencies. So, optimizing inspection planning of jacket structures needs for more accurate FDF value than the ones required by design.

This leads to use accurate data and fatigue computation. Accurate data on marine fouling includes:

on-site measurement,

- $\quad$ type of marine fouling: soft, hard. Species ranked in groups in accordance with hydrodynamic effect such as roughness. Such an approach is suggested in [10],

- distinction for vertical, diagonal and horizontal member with regard to flow direction, thickness as a function of water depth.

Thus, in the specific case of inspection planning or reassessment of existing platforms, real marine growth profiles rather than design code profiles are to be used. When optimization of inspection planning is aimed at, one must provide structure of colonization stochastic process in terms of species and thickness. It is still a challenge due to inspection cost and the great number of factors which affect marine growth process (salinity, access to light, temperature, ...). Thus two reasoning can be adopted:

a complete stochastic approach of marine growth process which allows to update safety indexes and optimize inspection and cleaning planning. Knowledge of stochastic process is actually not sufficient.

a reasoning on a given reference period where the aim is to analyze the state of structure after this time. Then an envelop of the marine growth thickness can be adopted.

This paper focuses on the second one.

\section{DYNAMIC AND FATIGUE BEHAVIOR : SPECTRAL ANALYSIS}

\section{Review of the basic principle of spectral analysis}

For fatigue computation, dynamic spectral fatigue analyses are used. At Bureau Veritas, the New Strudl Offshore software [NSO doc] is used. The main steps of this type of analysis is presented in the following paragraphs.

The main steps for dynamic spectral fatigue analysis are:

\section{modelling:}

- topside and jacket model: mass model and centre of gravity verification,
- hydrodynamic input: water added mass and marine growth mass generation,

- soil model: calculation of the equivalent stiffness matrix,

- model finalisation: substructure and connections model,

\section{ii. dynamic analysis:}

- mass model: computation of all masses,

- modal analysis: modes and natural periods calculations,

\section{iii. fatigue analysis:}

- sea states analysis: wave selection to model transfer functions,

- response calculation: stresses transfer function computation,

- scatter diagram: sea states distribution and wave spectra nput,

- damage: damage computation for the model,

- lifetime: computation of fatigue lifetime of each relevant tubular joint,

\section{v. cumulative damage computation:}

- in case of multi-phases or revamping project.

\section{Computational method}

\section{Dynamic analysis}

The general framework for dynamic analysis is classical dynamic equation:

$$
(M+M a) \ddot{x}(t)+C \dot{x}(t)+K x(t)=F(t)
$$

where $M$ is the structural mass matrix (including marine growth), $M a$ is the matrix of added water mass accounting for linear effects of hydro-elasticity, $C$ the damping matrix, and $K$ the structural stiffness matrix. $F$ is the nodal vector of forces imposed to the structure and $x$ is the nodal displacements vector.

For each eigen-frequency, the hot-spot stress can be computed, using stress concentration factors. The equation is solved using the classical modal superposition method.

\section{Fatigue analysis}

The fatigue analysis includes the following computational steps:

-wave spectra: defines the wave spectra for short-term sea states. The wave spectrum defines the density of probability of wave elevations.

-scatter diagrams: defines the probability that a given wave spectra occurs in a given wave heading. This is the long term distribution of the sea-states.

-transfer function: for a given wave with height $H$, period $T$, heading $\theta$ and phase $\varphi$, the hot-spot stress is computed. Then scanning over a set of phases gives the hot-spot stress range for the given hot-spot. Assuming that the hot-spot stress range is a linear function of the wave height, the normalised transfer function is computed. It gives the hot-spot stress range as a function of wave pulsation.

-response spectrum: using the normalised transfer function for hot-spot stress range and wave spectra, the response spectra is computed. 
-spectrum's damage integration: to simplify computations, the assumption of narrow banded spectrum is made. Thus, the density probability of the hot-spot stress range is the Rayleigh law. Then the relation-ship between the number of cycles to a given class of hot-spot stress ranges is established. For each wave spectra, the elementary damage can be computed.

-cumulated damage: using the scatter diagram and the elementary damages, the total damage for all hot-spots are computed.

\section{MAIN STUDY}

The aim here is to adopt a qualitative analysis (critical node ranking under fatigue loading) and a quantitative estimation of damage. Two studies are then suggested:

effect of selection of $C_{d}$ values deduced from API regulations and a complete probabilistic modeling. effect of profile selection based both on API regulations and on site measurements.

\section{Presentation of structure and site}

The structure considered here is a 4 legged jacket structure placed in warm sea with water depth of $80 \mathrm{~m}$. It is made up of about 1000 beams connected to 500 nodes including riser guides. The probability density of significant wave height whatever heading, presented on figure 1, is deduced from the scatter diagram based on Jonswap spectra. A Gumbel distribution is fitted.

\section{Selected marine growth profiles}

Three profiles are selected. A general profile 1 (see figure 2) is deduced from inspections on platform in the neighborhood of the studied platform. It is very close to default values suggested by API regulation (see figure 3) and is selected for a sensitivity study on hydrodynamics coefficients modeling. The profile 2 (see figure 3 ) is obtained by inspections on studied platform and aims to compare damage and ranking results to those obtained with API requirements. It can be seen that the growth thickness is about twice what it was observed on the neighboring platform. This randomness is well known in warm seas [9]. The third profile is the one given by API requirement for design (see figure 3 ).

\section{Introduction of marine growth effect on loading}

\section{API requirements}

API regulations [1] suggest two requirements for taking into account marine growth in structural analysis. The first is a standard profile of marine growth for taking into account screen effect and additional mass when computing environmental loading on structure. It is assumed a thickness of $50 \mathrm{~mm}$ in the upper 20 meters and zero for deeper waters. The second concerns hydrodynamic coefficients modeling. API suggests graphs in view to deduce values of drag coefficient $C_{d}$ depending on Keulegan-Carpenter number $K c$, assuming a post-critical flow running. In a first step, a steady-flow drag coefficient $C D S$ is deduced from relative roughness $e$, defined as the ratio between roughness and effective diameter. In a second step, drag coefficient comes from a graph linking $C_{d}$ and the ratio $K c / C D S$. Such values are called reference values in the following.

\section{Approach based on causality}

In reliability analyses, hydrodynamic coefficients are mainly considered as independent variables of environmental parameters [10]. From a probabilistic point of view, this assumption is not convenient but is certainly conservative as it allows a widest range of variable combination cases. Wolfram [11][12] suggest a global probabilistic modeling of hydrodynamic coefficients which allows to introduce both flow and experimental uncertainties. As the hydrodynamic coefficient modeling is of first importance [13], a finest approach is needed. The aim here is to find a causality relationship between environmental parameters (wave height $H$ and period $T)$ and hydrodynamic coefficients $\left(C_{d}, C_{x}, C_{x}{ }_{x}, C_{M}\right)$ through hydraulic parameters $R e$ (Reynolds number) and $K c$ (Keulegan-Carpenter number) which governs their main variations [2] and which are defined by equations (2):

$$
\operatorname{Re}=\frac{U^{*} D}{v} ; \quad K c=\frac{U^{*} T}{D} .
$$

where $U$ stands for the velocity intensity of the particles of the fluid, $T$ is the wave period, $v$ denotes the kinematics viscosity and $D$ the diameter of cylinders. Ones must underline that $D$ should be multiply by a variable $\theta_{m g}$ for taking into account the marine growth screen effects on beam diameter uncertainty. This variable has been shown [9] to be of second order importance when computing $R e$ and $K c$ if it is less than 10 percent of $D$. It is not considered here at this step of computations.

First let us remind why and where a probabilistic modeling of marine growth effects is needed for loading purposes. Sources of uncertainties are various. Firstly data, based on physical measurements using circumferential measurements, hand-held probes, scale bars or ROV's picture interpretations, leads to uncertain estimation of the thickness and the amount of coverage. Then force measurements in laboratory and signal processing lead to versatile results and to uncertain evaluation of loading. Finally, the use of Morison equations [14] for all orientations of beams of the jacket structure is an expansion of the standard case for which the formula as been developed. This leads us to introduce uncertainties in the model through the hydro-dynamic coefficients $C_{d} C_{x}, C_{x}{ }_{x}, C_{M}$, (respectively two drag and two inertia coefficients). The expression of Morison equations (3), translated in local coordinates (see figure 4), indicates that the force per unit span can be separated into drag and inertial components.

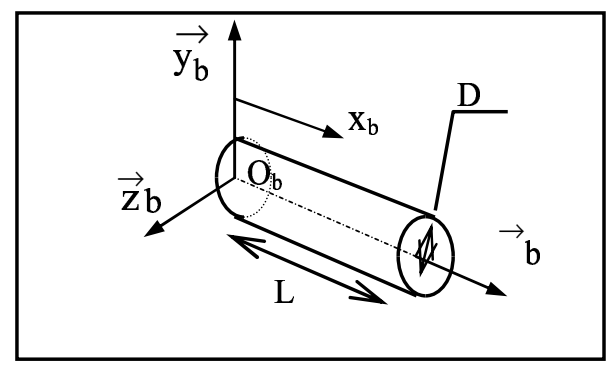

Figure 4. Local coordinates on a beam. 
$\vec{f}_{\mathrm{D}}(\mathrm{M})=\frac{1}{2} \rho \theta_{\mathrm{mg}} \mathrm{D}\left[\begin{array}{ccc}\pi \mathrm{C}_{\mathrm{x}} & 0 & 0 \\ 0 & \mathrm{C}_{\mathrm{D}} & 0 \\ 0 & 0 & \mathrm{C}_{\mathrm{D}}\end{array}\right] \overrightarrow{\mathrm{V}}(\mathrm{M})\|\overrightarrow{\mathrm{V}}(\mathrm{M})\|$
$\mathrm{f}_{\mathrm{I}}(\mathrm{M})=\frac{1}{4} \rho \theta_{\mathrm{mg}} \mathrm{D}^{2} \pi\left[\begin{array}{ccc}\mathrm{C}_{\mathrm{X}}^{\prime} & 0 & 0 \\ 0 & \mathrm{C}_{\mathrm{M}} & 0 \\ 0 & 0 & \mathrm{C}_{\mathrm{M}}\end{array}\right] \overrightarrow{\mathrm{A}}(\mathrm{M})$

where $\rho$ is the water density, $D$ the cylinder diameter, $\overrightarrow{\mathrm{V}}(\mathrm{M})$ and $\overrightarrow{\mathrm{A}}(\mathrm{M})$ are respectively the instantaneous flow velocity and the acceleration at point $M, \theta_{m g}$ acts as a multiplying coefficient for taking into account the marine growth screen effects on beam diameter uncertainty. Also, $\theta_{m g}$ depends on the marine growth thickness profile.

These equations generally predict quite well the main trends in measured data, once the appropriate joint distribution for hydro-dynamics coefficients, which depend on the sea-state parameters and the distribution of $\theta_{m g}$, can be provided. This factor itself depends on the marine growth nature (hard or soft) and its structure (percentage of coverage). Nevertheless Morison equations are not able to translate all the complexity of fluid-structure interaction with enough accuracy, such as high frequency content of the flow, gross vortex shedding effects, etc.

Keeping in mind these short comings as source of response problems, they are relevant to introduce non linear effects. Their complexity is consistent with respect to the large uncertainties in marine growth data or fluid interaction modeling. More complexity is not necessary suitable, and might be included in the model through relevant uncertainty variables. Thus, Morison equations have been used as basic model to develop a matrix response surface [16]. This response surface is based on a geometrical description of kinematics field $(\vec{V}(M), \vec{A}(M))$. It is shown to be very tractable for sensitivity studies. Such a response surface enables us to introduce the main uncertainties in flow modeling versus $C_{d}$ (drag coefficient) and also the increasing screen effects due to marine growth $\left(\theta_{m g}\right)$. Sensitivity studies have shown [15] that the variance of the coefficient $C_{d}$ contributes up to $61 \%$ to the variance of external nodal forces when the wave height and the corresponding period have influence ratios of $35 \%$ and $3 \%$ in the wave area. From a probabilistic point of view, these variables are not dependent. This paper proposes a conditioning of $C_{d}$ to the wave kinematics. By extension, a similar approach can be applied to the other hydrodynamic coefficients.

Using the response surface of kinematics field proposed in [12][15][16], there is a way to find a causality relationship between the waves parameters (wave height $H$ and period $T$ ) and the hydraulic parameters $R e$ and $K c$. In view to simplify the expression of probabilities, we adopt the notation:

$$
P\left(X_{i}\right)=P\left(x_{i}<X_{i}<x_{i+1}\right)
$$

According to uncertainties related to tests and the reliability of measurements, calculations are balanced by weights allocated to, depending on authors and expert judgment. For illustration purposes, let's consider 2 authors, $A 1$ and $A 2$. The expert will give weight according to the confidence he has in the data provided by each authors, namely $w 1$ and $w 2$. It leads to the probability:

$$
P\left(C d_{i}\right)={ }_{1} P_{A 1}\left(C d_{i} / K c_{i}\right) \cdot P\left(K c_{i}\right)+w_{2} P_{A 2}\left(C d_{i} / K c_{i}\right) \cdot P\left(K c_{i}\right)
$$

Then from (6), $P$ is a probability.

$$
P(C d)=\sum_{i=1}^{n} P\left(C d_{i}\right)=w \sum_{i=1}^{n} P_{A 1}\left(C d_{i}\right)+w 2 \sum_{i=1}^{n} P_{A 2}\left(C d_{i}\right)=1
$$

with $\quad w+w_{2}=1 . \mathrm{n}$ is selected such as $P\left(C d<C d_{1}\right)=0$ and $P\left(C d>C d_{n}\right)=0$.

\section{Semi probabilistic modeling}

Instead of using a complete probabilistic approach, the basic idea of the approach suggested in this paper is to define deterministic values of $C_{d}$ by a semi-probabilistic way. We work with trajectories of stochastic processes $R e$ and $K c$, deduced from stochastic process of water particle velocity $U$ which depends of fluctuations of basic variables $H$ and $T$. Then non linear relationship between $C_{d}$ and $R e$ or $K c$ are used to compute $C_{d}$ and to deduce the discrete probability density of $C_{d}$ (5). Finally the expected value of $C_{d}$ is used for deterministic calculations.

\section{RESULTS AND COMMENTS}

From NSO software, dynamic spectral analyses are performed. It allows to obtain two main outputs: node ranking and damage value. First, we analyze the effect on damage computing of the modeling described previously. Due to kinetics of water particle in the selected site, $K c$ is of first importance for assessment of $C_{d}$. Three values on $K c$ distribution are selected for $C_{d}$ and corresponding damage computing: minimum, mean and maximum value. Figure 5 shows iso-values curves of $K c$ in the plane (depth; abscissa in wave direction) for small vertical diameters $(0.1016 \mathrm{~m})$. This gives trajectories of stochastic process for the mean value of $K c$. Due to symmetry, only a quarter of the mean wave length (about 200 meters) is considered for range of abscissa. These mean values vary from 20 to 75 . Figure 6 presents damage computing obtained from these values and with reference (API) value of $C_{d}$. Effect of the selection of $K c$ value on damage can reach $15 \%$. In the following expected value of $C_{d}$ is selected. One must point out that the projection of velocity vector in plane perpendicular to the beam depends on angle between beam axis and flow direction, and several typologies should be studied. This point is not analyzed here and computations are made for vertical components only. Another geometrical characteristic affects flow regime and $C_{d}$ computation: the beam diameter (see equation (3)). Two classes of diameters are considered:

small one with diameter called $\operatorname{Dmin}$ between 0 and 0.5 with a great number of components of diameter 0.1016 .

large one with diameter called Dmax between 0.5 and 1.5 with a great number of components of diameter 1.2446.

Marine growth considered here is composed of hard fouling, mainly mussels with a percentage of cover which reaches $100 \%$. In this case, roughness is around $24 \mathrm{~mm} \mathrm{[17].}$ As the increase of diameter can reach $18 \mathrm{~cm}$, relative roughness 
$e$, stays in the range $[0.05 ; 0.2]$ for small diameters and $[0.015 ; 0.019]$ for large diameters.

\section{Numerical values of hydrodynamic coefficients obtained for simulations}

Both API regulation and semi-probabilistic modeling are used here. Let us first consider the flow regime. It has been shown [9] that $R e$ is not in post-critical range. For small cylinder it remains in range $\left[610^{3} ; 1.310^{4}\right]$ typical of critical at least sub-critical flow and for large one in the range $\left[7.410^{4}\right.$; $1.610^{5}$ ] typical of post-critical at least critical regime. Then API regulations cannot be used for all diameters in warm seas. Here values of $K c$ are less than 7 whatever diameter and depth (see figure 5 for small diameters).

For small diameters, the only source which allows to take into account such relative roughness in such regime is [18]: value of $e$ is 0.05 with constant values of $C_{d}$ in case of higher relative roughness, if $K c<10$; that is the case here. Theophanatos [17] suggests values of $C_{d}$ for such a relative roughness but in case of absolute roughness of $43 \mathrm{~mm}$. For large diameters, results presented in [17] are used.

Results of calculations concerning $C_{d}$ and $C_{M}$ are presented in table 1. Several ranges of water depth and two diameters for each are selected in view to take into account main variations in flow regime. It leads to variations among diameters from 1.2 to 1.6 (range $70-80 \mathrm{~m}$ ) and variations among depth for a given diameter from 1.2 to 1.62 (Dmax). For components above mean sea level, default values, called SubDmin and SubDmax, are selected.

\section{Sensitivity of fatigue results to $\mathrm{Cd}$ modelling}

We first focus on the node ranking from fatigue analysis considering the twelve most critical components. Effect of $C_{d}$ modeling is analyzed and the two approaches - i.e. API regulations (reference values), Probabilistic modeling (updated values) - are compared. Results are presented in graph on figure 7, with the marine growth profile plotted on figure 2. Except for the three most critical components, effect of $C_{d}$ modeling is important for ranking. If we consider now a quantitative analysis based on damage computing, results are presented on figure 8 . For some components, especially the most sensitive to fatigue behavior, fatigue Miner damages computed with reference values of $C_{d}$ are ten times lower than those suggested in this paper. Even updated $C_{d}$ are various there are linked with diameter and depth: this more accurate approach seems to be more conservative than API recommendations. This is due to the fact that sensitive components are mainly horizontal components for which updated $C_{d}$ are not suitable. An improved analysis should introduce more classes of diameter and orientation of component in the flow. In the following, updated values of $C_{d}$ are considered.

\section{Sensitivity of fatigue results to profile selection}

Let us consider now the profiles presented on figure 2 and compare the fatigue results. First, the rank of the critical tubular nodes is analyzed and the fourteen most sensitive to damage are presented on figure 9 . We can point out that despite a great discrepancy between shapes of marine growth profile (figures 2 and 3), the thirteen most sensitive are identical whatever the three profiles used in this paper. It is due to the fact that the tubular members are mainly horizontal and located in the splash zone. Second, marine growth thickness profile do not affect the rank of three most sensitive tubular connections (numbers 903, 957, 911). For the ten following connections, the profile shape do change the component rank not more than 2 places. In terms of qualitative analysis, for inspection planning for example, the profile suggested by API seems to be sufficient for close marine growth profiles.

If a quantitative analysis is required, for cleaning planning purpose for example, the figure 10 shows that the damage results are quite identical and that API leads to conservative results. This is due to tubular members which are at stake: they are in the splash zone where thickness suggested by API are 1.5 higher than those measured. Screen effect and corresponding loading and stresses are locally higher.

\section{Fatigue sensitivity ratio to marine growth}

In view to analyse sensitivity of damage to an increase or diminution of marine growth thickness, a fatigue sensitivity ratio to marine growth $(F R M)$ is introduced. $F R M(\tau)$ around a given thickness $\tau$ is defined by equation (7):

$$
\operatorname{FRM}(\tau)=-\frac{D(\tau+5)-D(\tau-5)}{10}
$$

where $D(\tau+/-5)$ denotes the damage for the current marine growth thickness plus/minus $5 \mathrm{~mm}$. This value of $5 \mathrm{~mm}$ represents $10 \%$ of the value proposed by the API. The damage around $\tau$ is only modified by screen effect because this level of thickness variation don't affect $C_{d}$ modeling. Results are presented on figure 11. They are consistent with those obtained previously: the three most sensitive tubular joints to fatigue loading have the lowest $F R M$ value. That is why they are not affected by the shape of the marine growth profile. For the other components, one must emphasize that some of them are not ranked as the most sensitive components to fatigue but are 5 or 7 times more sensitive than the first one in case of marine growth increasing.

This ratio $(F R M)$ is useful when looking at fatigue results of a given offshore platform, in view of the risk screening process for a Risk Based Inspection Planning study for example. Thus, critical joints from the fatigue point of view which show a high sensitivity to marine growth profile need extra attention to fatigue results.

\section{CONCLUSION}

As the marine growth affects mainly the flow around a structural component and its effective diameter, two analyses are suggested to observe the role of marine growth in fatigue computations.

Firstly it is shown that probabilistic modeling for hydrodynamics coefficients $\left(C_{d}\right.$ in the paper) is convenient and allows to introduce a semi-probabilistic approach. It is based on the response surface of stochastic process of water particle velocity which is introduced in Reynolds $(R e)$ and KeuleganCarpenters numbers $(K c)$ definitions. This approach introduces non-linear graphs giving $C_{d}$ versus $R e$ or $K c$ through a discrete probabilistic analysis. Then the use of discrete layers of water 
depth and classification of diameters is suggested. It is shown than future studies with a finest descriptions of diameters and effect of beam orientations is needed.

Secondly, the effect of the selection of the marine growth thickness profile is analyzed. It is shown that for the jacket platform of the study, the API requirements are to be conservative. For fatigue calculations, the main idea is to adopt an envelop of the real profile in the upper side of the platform where loading is dominant. A fatigue sensitivity ratio to marine growth $(F R M)$ is then introduced in view to analyze effect of an increase/decrease of marine growth thickness on damage. It seems to be a versatile aid-tool as it brings out the fact that some tubular joints have a convenient predicted damage but are very sensitive to an increase of marine growth thickness. As they are large uncertainties on the measurements of thickness, this result suggests to focus on sensitive components. Generally, inspected components are selected at given stages mainly from their accessibility.

\section{REFERENCES}

[1] API, (1993) «Recommended practice for planning, designing and constructing fixed offshore platforms Working stress design $»$, API recommended practice, $20^{\text {th }}$ Ed., pp. 113-119

[2] Schoefs F., Boukinda M., 2004, "Modelling of marine growth effect on offshore structures loading using kinematics field of water particle", Proc. $14^{\text {th }}$ I.S.O.P.E, 2328 May 2004, Toulon France, pp. 419-426.

[3] Faber M.H., Hansen P.F., Jepsen F.D. and Moller H.H. (2001) "Reliability-based management of marine fouling", Journal of Offshore Mechanics and Arctic Engineering, vol. 123, Issue 2, pp. 76-83, Elsevier Science Ltd.

[4] NORSOK Standard N-004, Rev. 2, October 2004, "Design of Steel Structures".

[5] Bureau Veritas Guidelines - RBI for structures, March 2005,"Risk based Inspection for steel fixed offshore platforms".

[6] Faber M.H., Engelund S., Sørensen J.D., Bloch A., 2000, "Simplified and generic risk based inspection planning". In Proceedings O.M.A.E 2000, paper no 6143.

[7] Rouhan A., Goyet J., Faber M.H., "Industrial implementation of risk based inspection planning - lessons learnt from experience-(2) the case of steel offshore structures", in Proceedings 23rd Offshore Mechanics and Arctic Engineering Conference, June 20-25, 2004, paper OMAE2004/S\&R-51573.

[8] Marine Technology Directorate Limited, 1992, "Appraisal of marine growth on offshore installations", MTD Limited Publication, 92/102, pp. 51.

[9] Boukinda M. and Schoefs F. (2004). "Statistical study of marine growth nature and process in Gulf of Guinea". Internal report $n^{\circ} 0410$ bio-01, Institut de Recherches en Génie Civil et Mécanique, University of Nantes.

[10] Engineering Committee on Oceanic Resources (1991), "Reliability analysis for offshore structures", pp.81.

[11] Wolfram J. Jusoh I. Sell D., (1993). "Uncertainty in the estimation of the fluid loading due to the effects of marine growth", Proc. 12 $2^{\text {th }}$ O.M.A.E, Glasgow, vol. II, pp. 219228.
[12] Wolfram J., Naguipour M. (1999). "On the estimation of Morison force coefficients and their predictive accuracy for very rough circular cylinders", Applied Ocean Research 21, pp. 311-328

[13] Schoefs F., "Sensitivity and uncertainty studies for the modelling of marine growth effect on offshore structures loading", Proc.21th OMAE, 23-28 June 2002, Oslo, Norway, Proceedings on CD, 7 pages (ASME 2002).

[14] Morison, J.R., O'Brien M.P., Johnson J.W. and Schaff. S.A. "The forces exerted by surfaces waves on piles", Petroleum trans. 1950.

[15] Schoefs F. (1996). "Response Surface of wave loading for reliability of marine structures", $\mathrm{PhD}$ Thesis, Nov. 1996, University of Nantes.

[16] Labeyrie, J. and Schoefs F., (1996). "Matrix Response Surfaces For Describing Environmental Loads", vol. II Safety and Reliability, Proc. of 15th OMAE, Florence, pp. 119-126.

[17] Theophanatos A. (1988). "Marine growth and hydrodynamic loading of offshore structures", $\mathrm{PhD}$ thesis, University of Strathclyde - UK.

[18] Gudmestad T.O., Moe G., (1996). "Hydrodynamic coefficients for calculation of hydrodynamic loads on offshore truss structures", Journal of Marine Structure 9, pp. 745-758. 


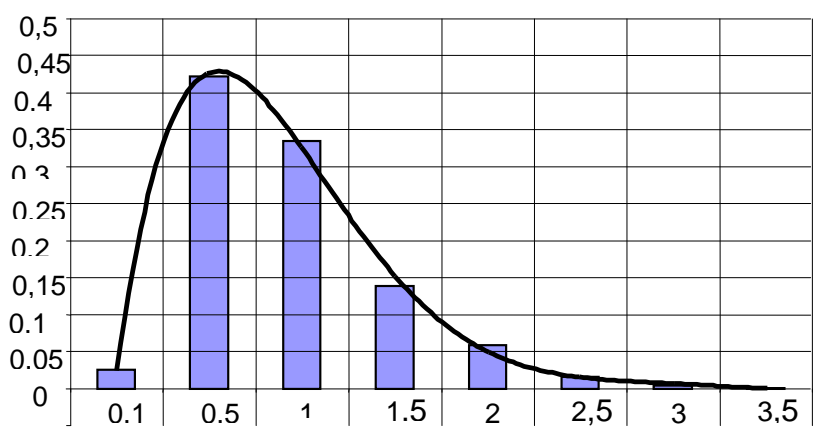

Figure 1. Distribution of significant wave height.

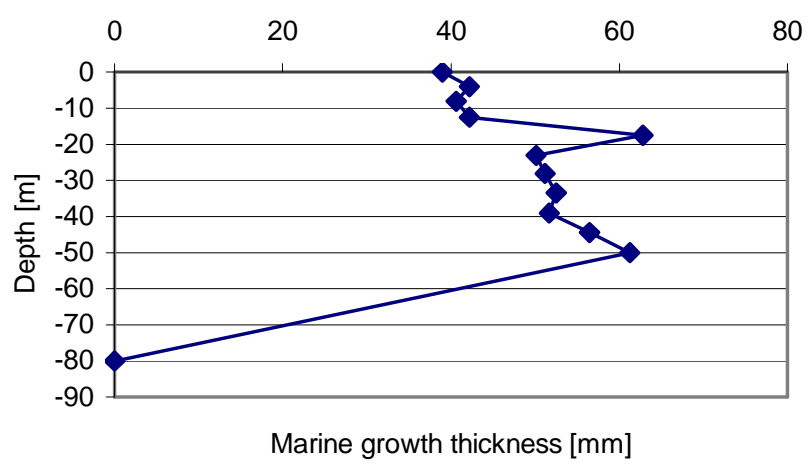

Figure 2. Profile 1 of thickness for a platform in the neighborhood of the studied platform.

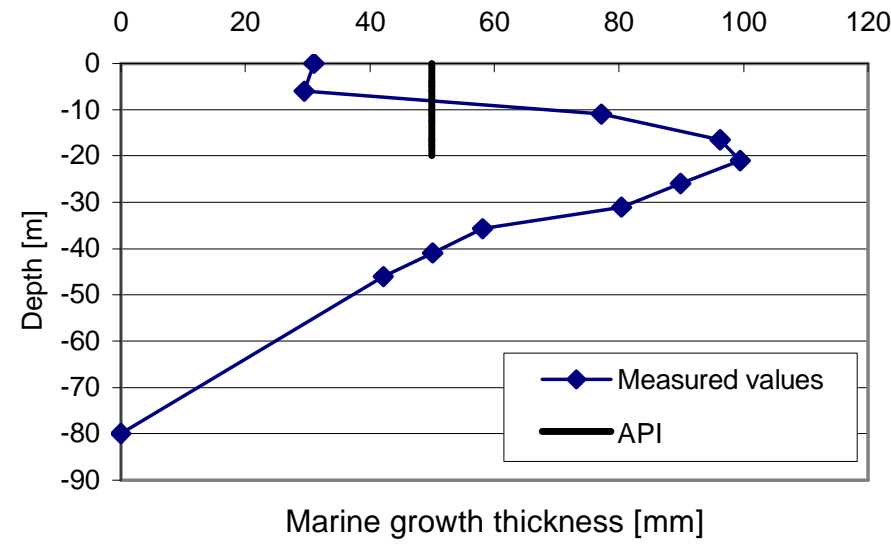

Figure 3. Profile 2 of thickness for the studied platform and profile 3 (API).

\begin{tabular}{|c|c|c|}
\hline $\begin{array}{c}\text { Depth range } \\
\text { and diameter class }\end{array}$ & $\begin{array}{c}\text { Drag } \\
\text { coefficient Cd }\end{array}$ & $\begin{array}{c}\text { Mass } \\
\text { coefficient } C_{M}\end{array}$ \\
\hline 00-20; Dmin & 1,6 & 2,5 \\
\hline 00-20; Dmax & 1,62 & 2,18 \\
\hline 20-40; Dmin & 1,6 & 2,5 \\
\hline 20-40; Dmax & 1,5 & 2,18 \\
\hline 40-50; Dmin & 1,6 & 2,5 \\
\hline 40-50; Dmax & 1,44 & 2,18 \\
\hline 50-70; Dmin & 1,6 & 2,5 \\
\hline 50-70; Dmax & 1,2 & 2,1 \\
\hline 70-80; Dmin & 1,6 & 2,5 \\
\hline 70-80; Dmax & 1,2 & 2,1 \\
\hline SubDmin & 0,5 & 2 \\
\hline SubDmax & 0,5 & 2 \\
\hline
\end{tabular}

Table 1. Profile 1 of thickness for a platform in the neighborhood of the studied platform.

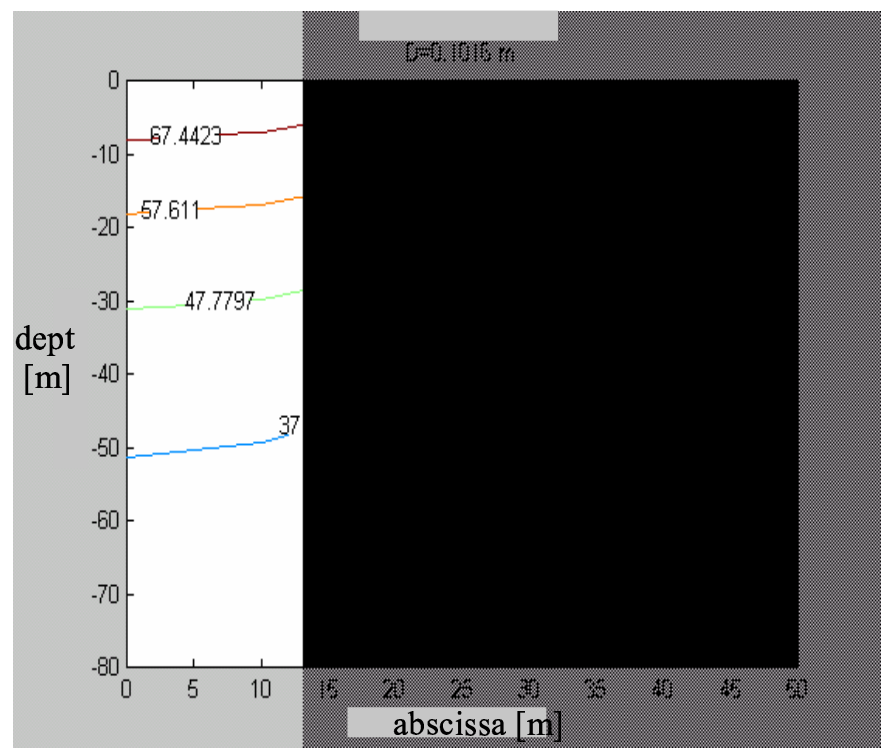

Figure 5. Iso-values of $\mathrm{Kc}\left(\mathrm{x}^{2} 0^{2}\right)$ with depth and abscissa in wave direction ; Dmin $=0.1016 \mathrm{~m}$. 


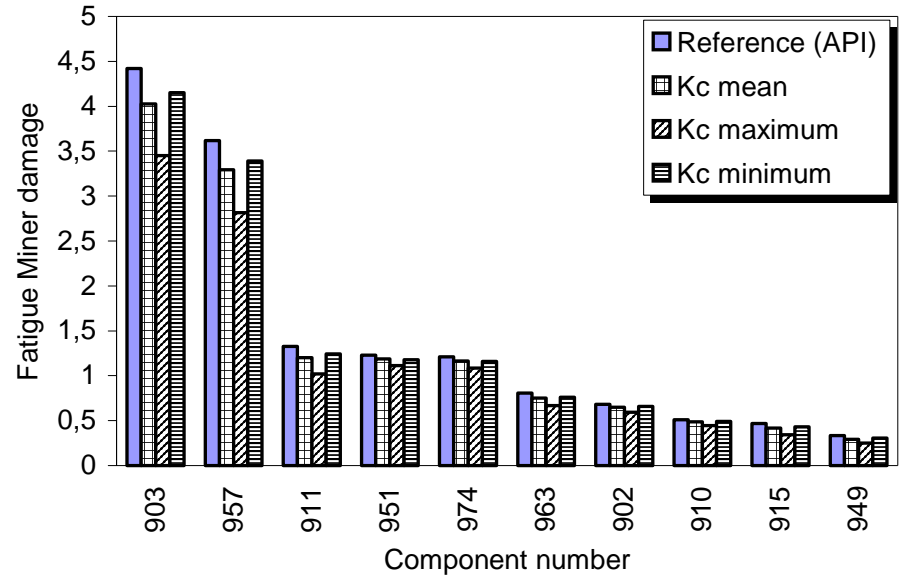

Figure 6. Effect of $\mathrm{Kc}$ on $\mathrm{Cd}$ modeling and resulting damage computation.

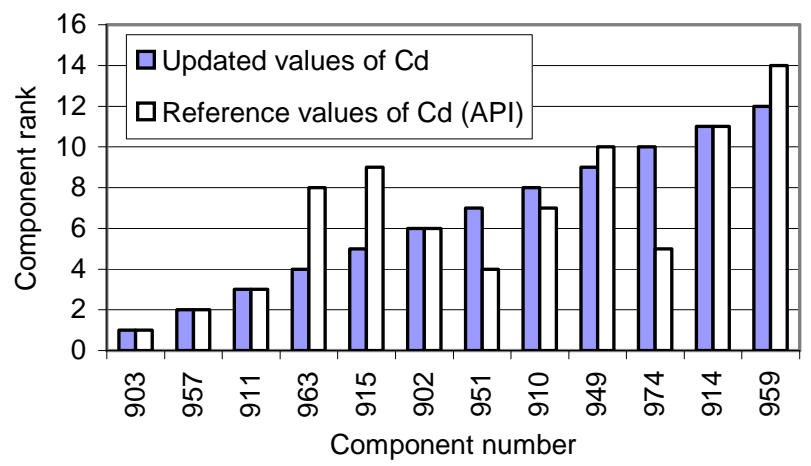

Figure 7. Effect of $\mathrm{Cd}$ modeling on ranking of the 12 most sensitive components for profile 1 .

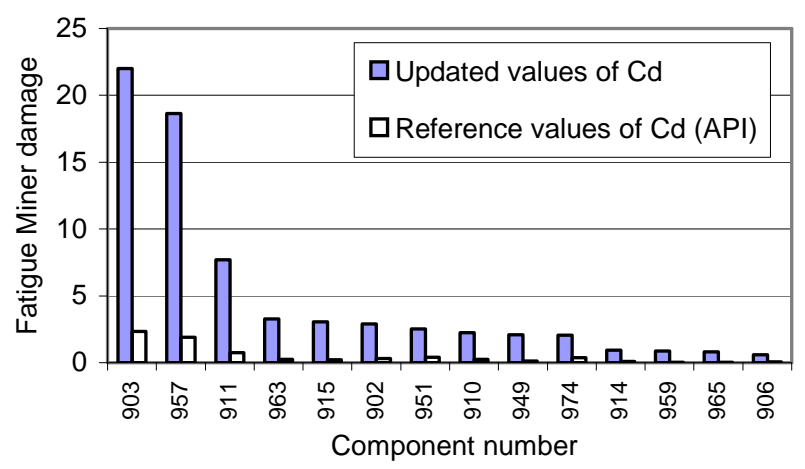

Figure 8. Effect of $\mathrm{Cd}$ modeling on damage computing of the 14 most sensitive components for profile 1.

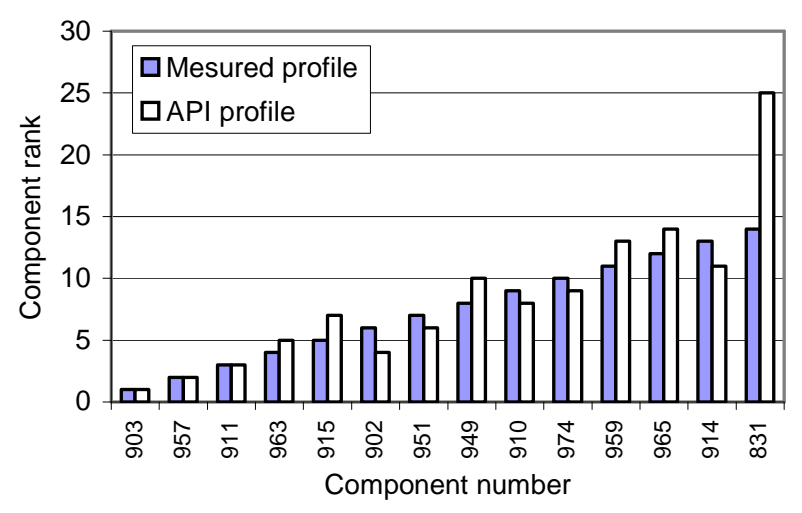

Figure 9. Effect of profile selection on ranking of the 14 most sensitive components.

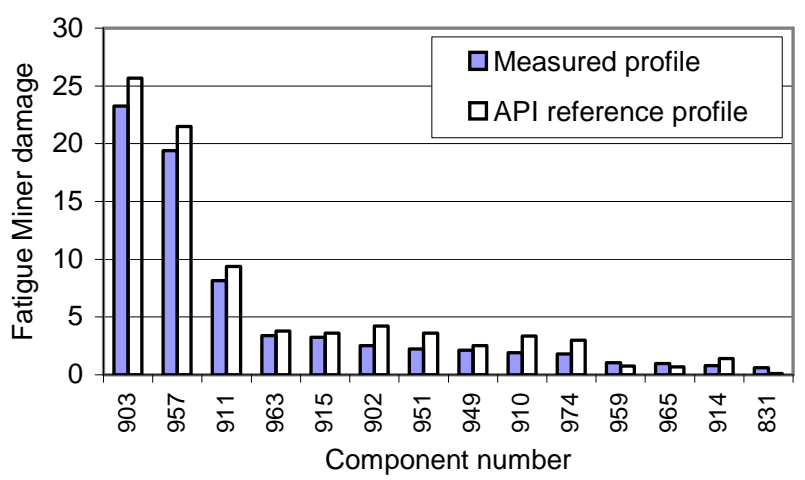

Figure 10. Effect of profile selection on damage computing of the 14 most sensitive components.

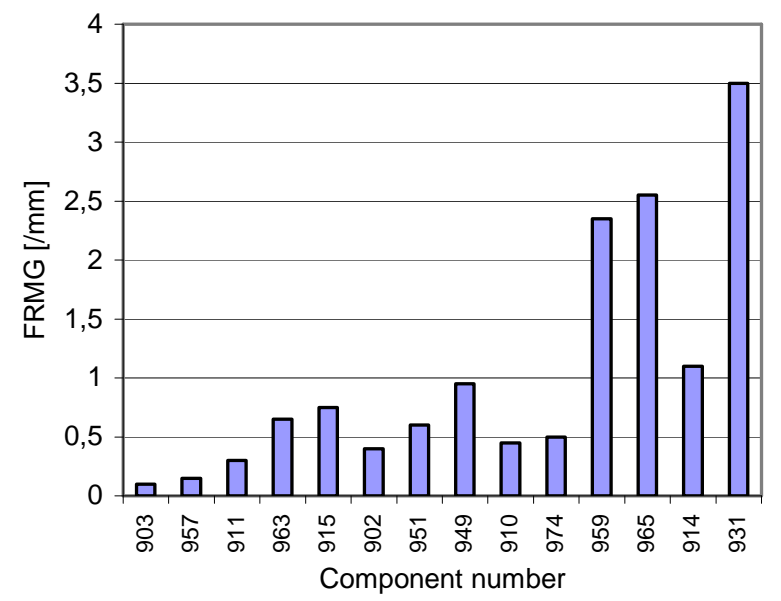

Figure 11. Fatigue sensitivity ratio to marine growth FRMG. 